\title{
INFLUÊNCIA DOS APLICATIVOS PARA CELULARES DE AFERIÇÃO DA OXIMETRIA E FREQUÊNCIA DE PULSO NA QUALIDADE DE VIDA DE PORTADORES DE DOENÇA PULMONAR CRÔNICA
}

\section{Heloisa Glass ${ }^{1}$, Gabriel Cordeiro Schimidt ${ }^{2}$, Igor Louza Pereira ${ }^{2}$, Paulo Henrique de Ramos Feitosa $^{3}$}

${ }^{1}$ Doutora, Docente da Escola Superior de Ciências da Saúde (ESCS) (heloisa.glass@escs.edu.br)

${ }^{2}$ Estudante de Medicina da Escola Superior de Ciências da Saúde (ESCS)

${ }^{3}$ Mestre, RTA da Unidade de Pneumologia do Hospital Regional da Asa Norte, Secretaria de Estado de Saúde do DF

DOI: 10.47094/IICNNESP.2021/143

\begin{abstract}
RESUMO
A OMS define "m-Health" como práticas médicas e de saúde pública dependentes de dispositivos móveis, especialmente os smartphones. Há grande esperança que este tipo de tecnologia irá traçar um novo cenário para as condutas de promoção de saúde. A escassez de estudos na literatura sobre a interface usuário e aplicativos relacionados à saúde dificulta compreender com acurácia esta relação. Principal objetivo desse trabalho é verificar a frequência de utilização de aplicativos relacionados a promoção da saúde pela população entrevistada e correlacionar a praticidade do método com a adesão do paciente com doença pulmonar crônica. O estudo é uma análise transversal, quantitativa. A amostra constituiu-se 47 pacientes do Hospital Regional da Asa Norte que preencheram um questionário composto por questões objetivas e incluiu perguntas relativas ao perfil dos entrevistados (idade, gênero, frequência de utilização de smartphone, quantidade de aplicativos relacionados a saúde que utiliza e adesão ao uso do celular para monitorar suas patologias). Os resultados apontaram o grande potencial de expansão e uso que os aplicativos relacionados à saúde podem ter, uma vez que a maioria das entrevistas possuem smartphones, acreditam nas melhorias na saúde trazidas pelos avanços tecnológicos e confiam em tecnologias móveis para desempenhar funções relacionadas ao cuidado em saúde. Os entrevistados acreditam no potencial de promoção de saúde de novas tecnologias como os M-Health apps, mas que ainda não as adotaram Supõe-se que algumas barreiras se impõem para que haja uma expansão da utilização dos apps, sendo necessário novas pesquisas sobre esses fatores. ALAVRAS-CHAVE: M-Health. Smartphone applications. Mobile Health. ÁREA TEMÁTICA: Outros.
\end{abstract}




\section{INTRODUÇÃO}

A OMS define "m-Health" como práticas médicas e de saúde pública dependentes de dispositivos móveis, especialmente os smartphones. A popularização de smartphones pelo mundo, incluindo o Brasil, tem trazido um novo paradigma para os cuidados em saúde. Acredita-se que os aplicativos dos celulares inteligentes (conhecidos também como apps) podem trazer grandes melhoramentos na promoção de saúde. ${ }^{1}$ Em 2016, o número total de apps da categoria m-Health nas maiores lojas de aplicativos era de aproximadamente 259 mil. $^{2}$ Com este grande número de pessoas com smartphones e o grande número de aplicativos relacionados à saúde, especula-se sobre o potencial impacto que estes apps podem exercer nos cuidados de saúde. ${ }^{3} \mathrm{~A}$ funcionalidades prometidas por estes aplicativos são múltiplas, como: monitoramento de doenças crônicas, lembretes para tomada de remédios, informação sobre remédios e/ou patologias etc. Entretanto, As atividades e funcionalidades mais acessadas nos smartphones não compreendem apps m-Health. ${ }^{4}$ Tal situação gera questionamentos sobre o uso ou não destes aplicativos por parte dos usuários; sobre possíveis barreiras para a não adoção destes apps como ferramenta suplementar na promoção de saúde. ${ }^{1}$

Na literatura científica, não há muitos artigos que explorem esta interface de usuários e m-Health apps; mas os poucos existentes buscam entender a validade e eficácia destes apps e se realmente podem trazer vantagens na promoção de saúde. Assim, este trabalho busca elucidar e enriquecer a literatura com mais análise de dados sobre a interface usuário e aplicativos/funcionalidades relacionadas à m-Health.

\section{METODOLOGIA}

O estudo é uma análise transversal, quantitativa. A amostra constituiu-se de pacientes (ou seu cuidador) do Hospital Regional da Asa Norte. Os critérios de inclusão para participação foram: pacientes, portadores de doenças pulmonares, que se voluntariarem a preencher o questionário. Para coleta de dados junto aos pacientes/cuidadores utilizamos de um instrumento padronizado com questões objetivas e escalas de opinião padronizadas. A análise descritiva dos dados foi feita com o Microsoft Excel. O questionário visa compreender o perfil dos participantes e mensurar a popularização dos aparelhos celulares inteligentes, compreendendo o perfil de uso dos que possuem smartphone, entender as principais funcionalidades e aplicativos utilizados pelos entrevistados, buscando delimitar o uso de aplicativos/funcionalidades relacionadas à saúde. Igualmente pretende-se investigar a opinião dos entrevistados sobre os benefícios que o avanço tecnológico para melhoramento da qualidade de vida e saúde; sobre a possibilidade de uso de tecnologias móveis, como smartphone, como meio de promoção de saúde e sobre a confiabilidade na acurácia de aferições (oximetria e frequência de pulso) por smartphones recentemente lançados. Ademais busca-se compreender a relevância da indicação por parte do profissional da saúde, como estímulo para adesão ao uso destes apps. Este questionário foi composto por questões objetivas e inclui perguntas relativas ao perfil dos entrevistados (idade, gênero, frequência de utilização de smartphone, quantidade de aplicativos relacionados a saúde que utiliza e adesão ao uso do celular para monitorar suas patologias) e perguntas sobre a opinião dos 
entrevistados utilizando escalas de concordância.

\section{RESULTADOS E DISCUSSÕES}

$\mathrm{Na}$ amostra de 47 entrevistados, 55,3\% são do sexo feminino. A figura 1 mostra distribuição por faixa etária. Observa-se que os partícipes da pesquisa se apresentam idade no intervalo de 30 até 89 anos, configura-se como o intervalo etário com boa quantidade de smartphones, conforme notado na PNAD 201855, sendo o número de homens e mulheres que possuem smartphone são próximos em

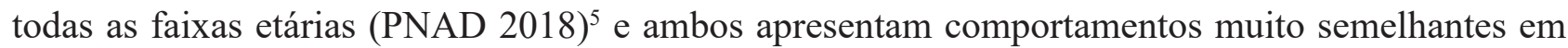
relação ao uso destes dispositivos (GMCS 2017). Dentre os entrevistados, apenas cinco afirmaram não possuir smartphone; evidenciando assim a aceitação dos smartphones como utensílio eletrônico essencial para a vida cotidiana, tendência observada na pesquisa realizada pela $\mathrm{FGV}^{-\mathrm{SP}^{6}}$, no início de 2018, apontando que há mais smartphones do que habitantes no Brasil. Diante deste quadro, a popularização do uso de aplicativos relacionados à saúde se torna algo factível.

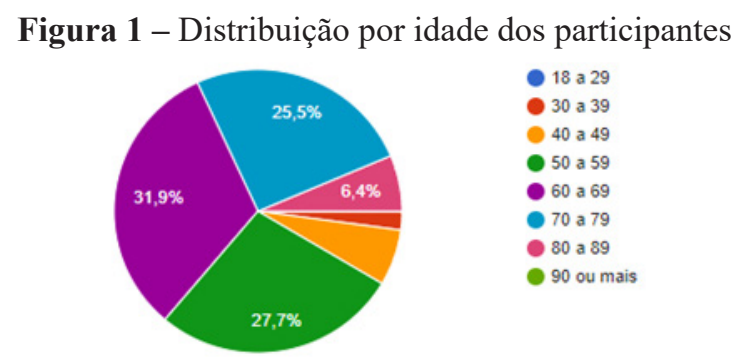

Na figura 2 temos o perfil de uso (frequência de uso semanal, frequência de uso diário, horas de uso diário) de smartphones. Os resultados encontrados são consoantes aos achados da pesquisa Global Mobile Consumer Survey $(2017)^{4}$, que aponta que a grande maioria das pessoas que possuem smartphones utilizam-no diariamente. Em relação ao tempo diário de uso, os resultados se aproximam dos achados da pesquisa realizada pelo "statista digital Market outdoor", que estimou uma média de 04 horas e 48 minutos de uso diário de smartphone pelo brasileiro. ${ }^{7}$ Compreendemos assim que os entrevistados em sua maior parte fazem uso rotineiro do smartphone e que estão familiarizados com a manipulação deste dispositivo tecnológico. 
Figura 2- Perfil de Uso de Celular

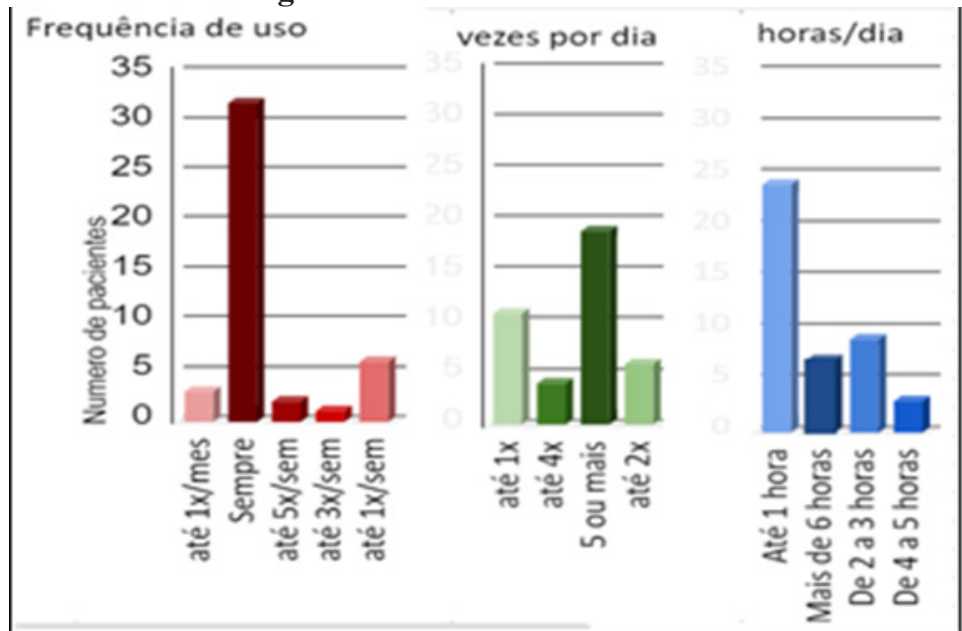

A figura 3 mostra as funções/utilidades mais usadas pelos entrevistados. Os resultados alcançados são semelhantes aos colhidos em pesquisa da PNAD (2018) e GMCS $(2017)^{4} 5$ mostrando que os conteúdos mais utilizados pelos usuários estão relacionados a comunicação (troca de mensagens por aplicativos ou e-mail, ligações) e uso de redes sociais. Apenas 9,1\% utilizam aplicativos da categoria M-Health. Aqui fica evidenciado que os entrevistados acreditam no potencial deste apps, mas poucos os utilizam.

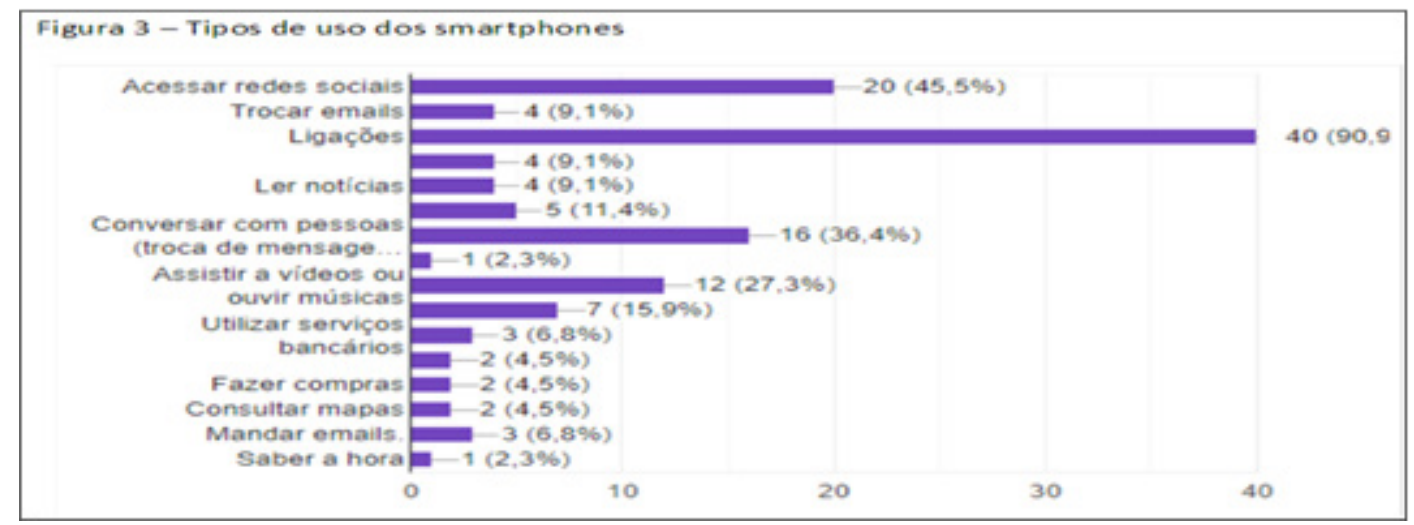

Em sua maioria, os entrevistados tendem a aceitar e aspiram novas ferramentas para melhor promoção de sua saúde, que podem ter uso mais específico e mais complexo, tal como triagem clínica e monitoramento de doenças (figura 4). Eles julgam que apps de saúde podem ter um papel na promoção de saúde e concordariam em usar estes apps, caso fossem indicados por um profissional de saúde. Isso expressa a confiança que os usuários têm nas sugestões destes profissionais de novas ferramentas para promoção de saúde. Indicando o papel importante que estes atores teriam. 0 nível de confiabilidade dos entrevistados em tecnologias tal como mensuração de oximetria de pulso e aferição de PA pelos celulares - quando comparados aos métodos mais tradicionais - é menor que no uso do celular como ferramenta de medição de sinais vitais. A razão para este achado não é única. Algumas barreiras se impõem para que haja uma expansão da utilização dos apps. Muitos pacientes desconhecem a categoria de aplicativos móveis relacionados à saúde. ${ }^{3}$ Além disso, fica claro que o uso dessas ferramentas está condicionado a sugestão por parte da equipe de saúde. E as equipes ainde 
esperam que haja uma melhor normatização, "Guidelines" que trouxessem recomendações claras quanto o que deve contemplar e no que se deve basear os aplicativos m-Health. ${ }^{9}$

Figura 4: Opinião dos entrevistados sobre a utilidade, confiabilidade e acessibilidade de aplicativos de celular e das novas tecnologias.

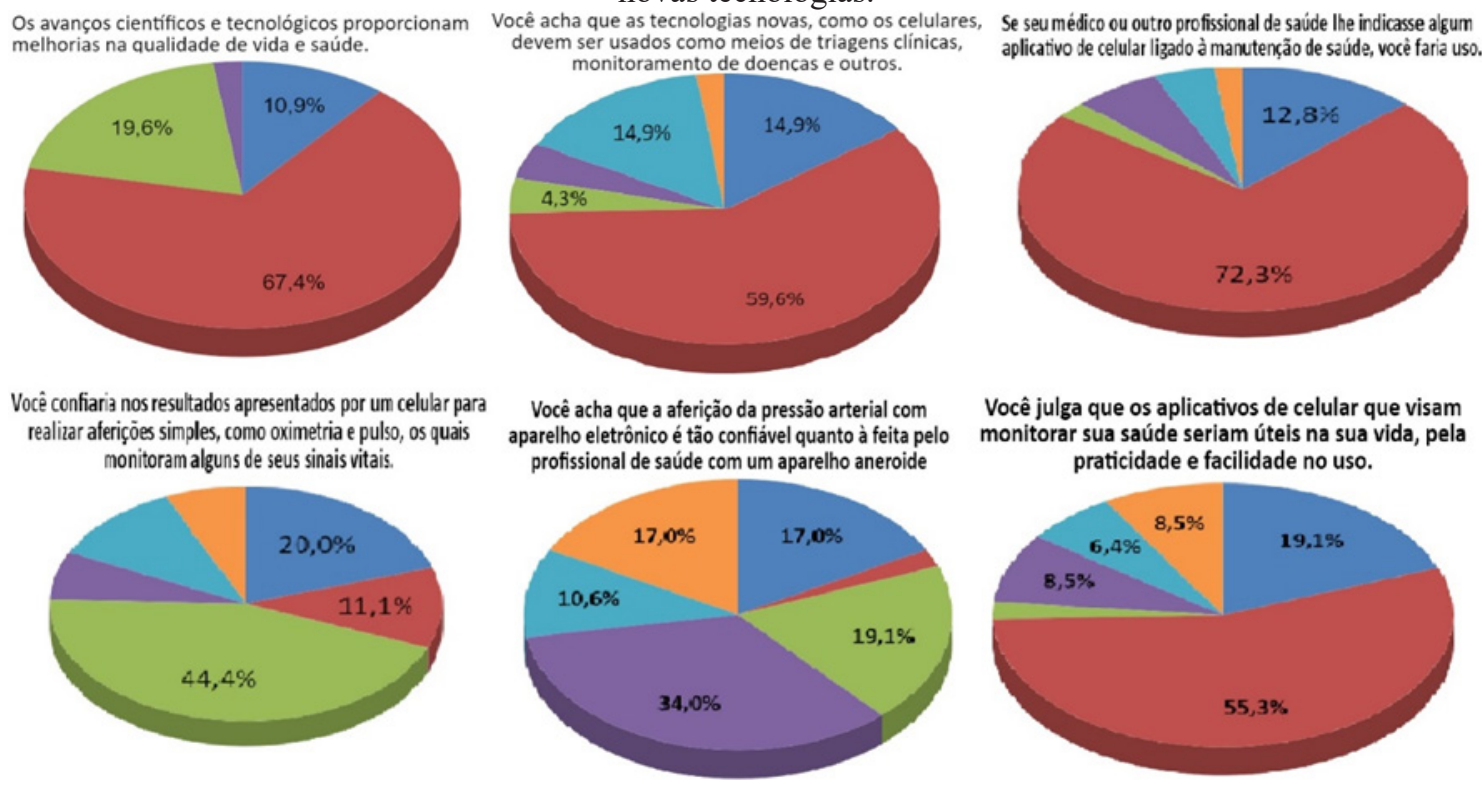

- Concordo acentuadamente Concordo totalmente Discordo pouco Concordo pouco Discordo totalmente Discordo acentuadamente

\section{CONCLUSÕES}

Os entrevistados acreditam no potencial de promoção de saúde de novas tecnologias como os M-Health apps, ainda não adotaram uso destes para promoção de sua própria saúde, mas o fariam se indicado por profissional de saúde.

\section{PRINCIPAIS REFERÊNCIAS}

1. HAS FRANCE. Assessment and Improvement of Practice Good Practice Guidelines on Health Apps and Smart Devices (Mobile Health or mHealth). [s. 1.], n. October, 2016. Disponível em: https://www.has-sante.fr/portail/upload/docs/application/pdf/2017-03/dir1/good_practice_ guidelines_on_health_apps_and_smart_devices_mobile_health_or_mhealth.pdf

2. SAADATFARD, O and ÅRSAND, E. M-health apps by numbers. Norwegian centre for health research. Fact sheets, [s. 1.], v. 3, n. 3, 2016.

3. HEFFERNAN, Kayla Joanne et al. Guidelines and Recommendations for Developing Interactive eHealth Apps for Complex Messaging in Health Promotion. JMIR mHealth and uHealth, [s. 1.], v. 4, n. 1, p. e14, 2016. Disponível em: http://mhealth.jmir.org/2016/1/e14/

4. DELOITTE. Global Mobile Consumer Survey 2014. [s. 1.], p. 1-29, 2017. Disponível em: http:// www2.deloitte.com/be/en.html 
5. TOBERGTE, David R.; CURTIS, Shirley. Acesso à Internet e à Televisão e Posse de Telefone Móvel Celular para Uso Pessoal. Journal of Chemical Information and Modeling, [s. 1.], v. 53, 1 n. 9, p. 1689-1699, 2013. VAN VELSEN, Lex; BEAUJEAN, Desirée Jma;

6. MEIRELleS, Fernando S. 29a Pesquisa Anual, 2018 Administração e Uso da TI nas Empresas. [s. 1.], 2018. Disponível em: https://eaesp.fgv.br/sites/eaesp.fgv.br/files/pesti2018gvciappt.pdf

7. MARTIN ARMSTRONG. Smartphone Addiction Tightens Its Global Grip Statista Digital Market Outdoor, , 2017. Disponível em: https:/www.statista.com/chart/9539/smartphone-addictiontightens-its-global-grip/

8. NEWBOLD, Tony. What do patients and carers need in health apps - but are not getting ? [s. 1.], n. November, 2014..

9. AlBRECHT, U. V. Chances and Risks of Mobile Health Apps (CHARISMHA). [s. 1.], p. 14-41, 2016. Disponível em: https://www.bundesgesundheitsministerium.de/fileadmin/Dateien/3 Downloads/A/App-Studie/charismha_abr_v.01.1e-20160606.pdf 